\title{
Sobre condiciones suficientes de estabilidad para sistemas mecánicos sometidos a fuerzas dependientes de la velocidad por el método directo de Liapunov
}

RAFAEL RODRIGUEZ REBOLLO

Dr. Ingeniero de Caminos, Canales y Puertos

\begin{abstract}
sinopsis
Consideramos la estabilidad de un estado de un sistema mecánico, discreto o continuo, sometido a fuerzas generalizadas dependientes o no de la velocidad y cuyas ecuaciones variacionales de movimiento, para pequeñas perturbaciones, son ecuaciones diferenciales de segundo orden respecto del tiempo y de una estructura formal frecuente en diversos problemas de estabilidad de medios continuos y de sistede una estructura formal frecuente en diver
mas de particulas y de sólidos rígidos.

Se obtienen criterios de estabilidad y de estabilidad asintótica según Liapunov por el método directo, generalizando resultados conocidos. Son condiciones de estabilidad condicional para soluciones de tipo exponencial respecto del tiempo.
\end{abstract}

\section{Introducción}

La problemática de la estabilidad de estados mecánicos de un sistema ocupa un lugar de primordial importancia en la temática de la mecánica, tanto teórica como aplicada, y ha sido motivo de continuados estudios desde Arquímedes hasta nuestros días.

En el estudio de la estabilidad de una posición de equilibrio o de un movimiento determinado de un sistema mecánico es frecuente el uso del método de las pequeñas perturbaciones. Las ecuaciones resultantes, expresadas con operadores adecuados, son formalmente idénticas en numerosas ocasiones en el estudio de diversos sistemas físicos.

Para sistemas de partículas y de sólidos rígidos, en dichas ecuaciones operacionales aparecen términos constituidos por matrices simétricas o antisimétricas, con elementos reales, aplicadas sobre los vectores incremento de velocidad y desplazamiento. Ejemplos pueden verse en diversos tratados [1].

Así mismo, con cierta frecuencia, las ecuaciones variacionales de movimiento, en la aproximación lineal, para sólidos elásticos, plasmas y fluidos, presenta la misma estructura en forma operacional, pero con operadores diferenciales lineales hermíticos o antihermíticos, con elementos reales en lugar de las matrices.

Recientemente, R. J. Knops and E. W. Wilkes [2] han desarrollado una teoría general rigurosa de estabilidad según Liapunov, con especial atención al método directo o segundo método, que aplican fundamentalmente a sólidos elásticos. Incluyen el estudio de la estabilidad para ciertas ecuaciones variacionales de movimiento, en forma operacional.

Otros autores, como Dickerson, J. R. y Caughey, T. K. [3] han analizado también la estabilidad para otros tipos de ecuaciones operacionales.

Por otra parte, en numerosos problemas, resulta útil la consideración de soluciones producto de una función del tiempo y otra del vector posición. Citamos, entre otros muchos, a Leipholtz [4].

Nuestro propósito en el presente trabajo es la obtención de criterios de estabilidad según Liapunov de estados de ciertos sistemas mecánicos discretos o continuos, de forma unificada, mediante la aplicación del método directo a la ecuación de movimiento que obedecen, expresada en forma operacional. Consideramos soluciones exponenciales en el tiempo, obteniendo criterios de estabilidad condicional. 


\section{Formulación general}

Consideramos un sistema mecánico, inicialmente en una configuración de referencia $C_{0}$, con un volumen material $V_{0}$, limitado por una superficie material $S_{0}$. La aplicación de las fuerzas conduce a una configuración original $\circ$ no perturbada $C$, de la que se estudian las propiedades de estabilidad. Nos referimos a un sistema fijo único de coordenadas cartesianas rectangulares.

Estudiamos la estabilidad según Liapunov de un estado de dicho sistema mecánico, cuando las ecuaciones variacionales de movimiento, para pequeñas perturbaciones respecto a la configuración $C$, se escriben de forma general como sigue:

$$
\ddot{\vec{u}}+2 \hat{E} \vec{u}+\hat{D} \vec{u}=\overrightarrow{0}
$$

donde el punto superpuesto representa la derivada con respecto al tiempo.

Las condiciones de contorno son:

$$
\widehat{R u}=\overrightarrow{0}
$$

La configuración $C$ corresponde a la solución nula:

$$
u_{i}(\vec{x}, t)=0, i=1,2,3
$$

donde $\vec{x}$ representa el vector posición en $V$.

En el caso general, $\hat{E}, \hat{D}$ y $\hat{R}$ son operadores lineales diferenciales sobre $\vec{x}$, con coeficientes reales constantes. La ecuación (1) es entonces válida en numerosos problemas de medios continuos, en particular de sólidos elásticos.

Consideramos el conjunto $X$ formado por todas las funciones vectoriales definidas en $V$ y que satisfacen las condiciones de contorno (1)'.

Para $T$ el semigrupo de todos los números reales no negativos, el sistema dinámico asociado $(X, T)$ está formado por todas las funciones vectoriales $u_{i}$ definidas en $(0, \chi)$, tomando valores en $X$ y tales que satisfacen las ecuaciones variacionales de movimiento (1). Las soluciones estables constituyen un subconjunto de $X$.

Podemos considerar, por simplicidad, que las soluciones estables son excepcionales del tipo periódico puro, respecto del tiempo $t$, o bien exponencialmente decrecientes con $t$ para la estabilidad asintótica. Estas soluciones constituirán un espacio $P, P \subset X$, cuyos elementos serán designades:

$$
\vec{u}=\vec{f}(\vec{x}) e^{(i \alpha-\beta) t}
$$

donde $\alpha$ y $\beta$ son reales, $\beta \geqslant 0$ y $\vec{f}$ es un vector columna, cuyas componentes son funciones de $\vec{x}$.

$\mathrm{Si}$ todas las soluciones son estables pertenecientes a $P$ el estado considerado es estable según Liapunov en la aproximación lineal. Es un resultado de estabilidad condicional.

Se define el producto interno como sigue:

$$
\overrightarrow{(\eta}, \vec{\xi})=\int_{v(t)} \vec{\eta}^{*} \cdot \vec{\xi} d V, \vec{\eta}, \vec{\xi} \in P
$$

donde el asterisco indica "complejo conjugado", siendo la integral finita.

La norma de $\vec{\eta} \in P$ es:

$$
(\vec{\eta}, \vec{\eta})=\int_{v(t)} \vec{\eta}^{*} \cdot \vec{\eta} d V
$$


En el caso general, podemos escribir:

$$
\begin{aligned}
& \hat{E}=\hat{E}_{1}+i \hat{E}_{2}=\hat{E}^{s}+\hat{E}^{a} \\
& \hat{D}=\hat{D}_{1}+i \hat{D}_{2}=\hat{D}^{s}+\hat{D}^{a}
\end{aligned}
$$

donde el superındice «s» corresponde a operadores hermíticos y el «a», a operadores antihermíticos.

Cuando $\hat{E}=\hat{E}^{s}$, siendo $\hat{E}^{s}$ un operador hermítico positivo, el término $2 \hat{E}^{s} \dot{\vec{u}}$ de (1) constituye un caso generalizado de fuerzas disipativas y cuando $\hat{E}=$ $=\hat{E}^{a}$, de fuerzas giroscópicas. $\bar{D}=\bar{D}^{s}$ corresponde a fuerzas derivables de una función potencial.

La ecuación operacional (1) es también válida en diversos problemas de estabilidad lineal de estados de sistemas mecánicos discretos, de partículas y de sólidos rígidos, en cuyo caso $\vec{u}$ es un vector en un espacio euclídeo real de dimensiones el número de grados de libertad del sistema y los operadores son matrices simétricas y antisimétricas, con elementos reales. El producto interno será $(\vec{u}, \vec{v})=\vec{u}^{*} \cdot \vec{v}$, como es usual.

\section{Condiciones suficientes de estabilidad}

El problema puede interpretarse como el de una partícula de masa unidad sometida a las fuerzas $-\hat{D} \vec{u}$ y $-2 \hat{E} \vec{u}$ por unidad de volumen.

En el caso general, $\hat{D}=\hat{D}^{s}+\hat{D}^{a}$ y $\hat{E}=\hat{E}^{s}+\hat{E}^{a}$, donde $\hat{D}=\hat{D}_{0}^{s}+\hat{D}_{1}^{s}$, correspondiendo el operador hermítico $\hat{D}_{0}^{s}$ a fuerzas conservativas y el operador $\hat{D}_{1}$ $=\hat{D}_{1}^{s}+D^{a}$ a fuerzas no conservativas generales.

La energía cinética $T$ resulta ser:

$$
T=\left.\frac{1}{2} \int_{v(t)} \frac{\partial \vec{u}}{\partial t}\right|^{2} d V=\frac{1}{2}(\dot{\vec{u}}, \ddot{\vec{u}})
$$

y definimos la energía potencial $U$ como:

$$
U=\frac{1}{2}\left(\vec{u}, \hat{D_{0}^{s}} \vec{u}\right)
$$

Consideramos las soluciones de la forma $\vec{u}=\vec{\eta}=\vec{f}(\vec{x}) e^{i \omega t}$

$$
\text { donde } \omega=\alpha+i \beta, \alpha \text { y } \beta
$$

reales, $\beta \geqslant 0$. Sustituyendo $\vec{u}=\vec{\eta}$ en (1), obtenemos:

$$
\ddot{\vec{\eta}}=-\left(\hat{G}^{s}+\hat{H}^{a}\right) \vec{\eta}=-\hat{L \eta}
$$

donde

$$
\begin{gathered}
\hat{G}^{s}=\hat{G}_{1}^{s}+\hat{G}_{2}^{s}, \hat{H}^{a}=\hat{H}_{1}^{a}+\hat{H}_{2}^{a} \\
\hat{G}_{1}^{s}=\hat{D}_{0}^{s}+\hat{D}_{1}^{s}=\hat{D}^{s}-2 \beta \hat{E}^{s} \\
\hat{G}_{2}^{s}=2 \alpha i \hat{E}^{a} \\
\hat{H}_{1}^{a}=\hat{D}^{a}-2 \beta \hat{E}^{a} \\
\hat{H}_{2}^{a}=2 \alpha i \hat{E}^{s}
\end{gathered}
$$

Observemos, en efecto, que el operador $i \hat{E}^{a}$, es hermítico:

$$
\left(\vec{\eta}, i \hat{E}^{a} \vec{\xi}\right)=i\left(\vec{\eta}, \hat{E^{a} \vec{\xi}}\right)=-i\left(\vec{E}^{a} \vec{\eta}, \vec{\xi}\right)=\left(i \hat{E^{a}} \vec{\eta}, \vec{\xi}\right)
$$

así como el operador $i \hat{E}^{s}$ es antihermítico. 
Fundamentalmente, consideramos dos casos.

I. Las fuerzas que actúan sobre el sistema son conservativas y giroscópicas: $\hat{E}=\hat{E}^{a}$ y $D=\hat{D}^{s}=\hat{D}_{0}^{s}$.

Consideremos las soluciones $\vec{u}=\vec{\eta}$ para $\beta=0$.

Adoptamos el potencial generalizado $U_{1}$ :

$$
U_{1}=\frac{1}{2}\left(\vec{\eta}, 2 \hat{E^{a} \dot{\eta}}+\hat{D s} \overrightarrow{s \eta}\right)=\frac{1}{2}\left(\vec{\eta}, \hat{G^{s} \eta}\right)
$$

habida cuenta de que $\dot{\vec{\eta}}=i \vec{\eta}$ y de que $i \hat{E}^{a}$ es un operador hermítico.

Tenemos:

$$
\begin{gathered}
\overline{T+U_{1}}=\frac{1}{2}(\ddot{\vec{\eta}}, \dot{\vec{\eta}})+\frac{1}{2}(\dot{\vec{\eta}}, \ddot{\vec{\eta}})+\frac{1}{2}\left(\overrightarrow{\left.\vec{\eta}, \dot{\hat{G}^{s}} \vec{\eta}\right)}=0\right. \\
T+U_{1}=h=\text { cte. }
\end{gathered}
$$

Obtenemos la expresión de la integral de la energía del sistema para el potencial $U_{1}$.

Condición suficiente de estabilidad según Liapunov, respecto a las medidas $\rho_{0}=T(0)+U_{1}(0)$ y $\rho=(\vec{\eta}, \vec{\eta})$

es:

$$
\left(\vec{\eta}, 2 \hat{E^{a}} \dot{\vec{\eta}}+\hat{D}_{0}^{s} \vec{\eta}\right) \geqslant c(\vec{\eta}, \vec{\eta})
$$

donde $c$ es una constante positiva, para todo posible $\vec{\eta}$.

El resultado anterior expresa que la presencia de fuerzas giroscópicas, en el caso considerado, es equivalente a la introducción de un potencial giroscópico tal que existe estabilidad cuando la forma asociada al potencial total es definida positiva en el sentido de (4). No es necesario que el potencial de las fuerzas conservativas tenga un mínimo, equivalente a que el operador $\hat{D}^{s}=\hat{D}_{0}^{s}$ sea hermítico positivo, sino que es suficiente que $\hat{D}_{0}^{s}+2 \alpha i \hat{E}^{a}$ sea un operador hermítico positivo para los posibles $\alpha$. Es un efecto de estabilización de las fuerzas giroscópicas generalizadas, en presencia de fuerzas conservativas generalizadas.

El resultado obtenido es válido para sistemas discretos y continuos que obedecen la ecuación operacional (1).

II. Caso general, con $\hat{E}=\hat{E}^{s}+\hat{E}^{a}$ y $\hat{D}=\hat{D}_{0}^{s}+\hat{D}_{1}^{s}+\hat{D}^{\mathrm{a}}=\hat{D}^{\mathrm{s}}+\hat{D}^{\mathrm{a}}$.

Consideramos las soluciones $\vec{u}=\vec{\eta}$ para $\beta \geqslant 0$. Si $\beta=0$ existe estabilidad y si $\beta>0$, estabilidad asintótica. El valor nulo de $\beta$ representa la frontera de separación de las soluciones estables asıntóticamente de las inestables exponencialmente crecientes.

Observemos que el valor nulo de $\beta$ en la ecuación operacional (2) no suprime la presencia de los operadores $\hat{D}^{s}, \hat{E}^{s}, \hat{D}^{\mathrm{a}}$ y $\hat{E}^{\mathrm{a}}$.

Ila. Sea de nuevo la ecuación (2):

$$
\ddot{\vec{\eta}} \rightarrow \hat{L \eta}=\overrightarrow{0}
$$

Supongamos que $\hat{M}^{s}$ y $\hat{N}^{s}$ son operadores tales que

$$
\hat{L \eta}=\hat{M} s \vec{\eta}+i \hat{N} \vec{\eta}
$$

Consideramos la funcional de Liapunov:

$$
\left.U=\frac{1}{2} \vec{\eta}, \hat{M}^{s} \vec{\eta}\right)
$$


y vemos que:

$$
\dot{T}+\dot{U}=-\alpha\left(\vec{\eta} \quad \hat{N}^{s} \vec{\eta}\right)
$$

Condiciones suficientes de estabilidad, respecto a las medidas $\rho_{0}=T(0)+$ $+U(0)$ y $\rho(\eta, \eta)$, son

$$
\begin{gathered}
\operatorname{Re}(\vec{\eta}, \overrightarrow{L \eta}) \geqslant c(\vec{\eta}, \vec{\eta}) \\
-\alpha \operatorname{Im}(\vec{\eta},[\overrightarrow{\eta \eta}) \leqslant 0
\end{gathered}
$$

para una constante positiva $c$ y todo posible $\vec{\eta}$. «Re» y «Im» indican «parte real" y "parte imaginaria", respectivamente.

Ilb. Otras condiciones suficientes de estabilidad se obtienen también como sigue, con ciertas restricciones sobre los operadores. Consideramos las medidas $\rho_{0}$ y $\rho$ anteriores.

Adoptamos el potencial generalizado:

$$
\begin{gathered}
U=\frac{1}{2} \overrightarrow{\eta \eta},\left(k_{1} \hat{D^{s}}+2 \alpha i k_{2} \hat{E}^{a}-2 \beta k_{3} \hat{E}^{s}+i k_{4} \hat{D}^{a}-\right. \\
\left.\left.-2 \beta k_{5} i \hat{E}^{a}-2 \alpha k_{6} \hat{E}^{s}\right) \vec{\eta}\right],
\end{gathered}
$$

donde las cantidades $k_{i}$ son números reales.

El cálculo de $\dot{T}+\dot{U}$ nos proporciona la igualdad:

$$
\begin{aligned}
\dot{T}+\dot{U}=\{ & \left\{\eta, \quad\left[\beta\left(1-k_{1}\right) \hat{D}^{s}+2 \beta\left(1-k_{2}\right) \alpha i \hat{E}^{a}-2 \beta^{2}\left(1-k_{3}\right) \hat{E}^{s}+\right.\right. \\
& \left.\left.+\alpha i \hat{H}^{a}-\beta k_{4} i \hat{D}^{a}-2 \beta^{2} k_{5} i \hat{E}^{a}+2 \alpha \beta k_{6} \hat{E}^{s}\right] \vec{\eta}\right\}
\end{aligned}
$$

Condiciones suficientes de estabilidad son:

$$
\begin{gathered}
U \geqslant c(\vec{\eta}, \vec{\eta}) \\
\dot{T}+\dot{U} \leqslant 0
\end{gathered}
$$

donde $c$ es una constante positiva, para todo posible $\vec{\eta}$.

Si consideramos la expresión anterior para $U$ y damos el valor $K$ a las constantes $K_{i}$ no nulas, podremos escribir las siguientes condiciones suficientes de estabilidad:

$$
k\left\{\vec{\eta}, \quad\left[\text { Parte } \hat{G}^{s}+\text { Parte }\left(i \hat{H}^{a}\right)\right] \vec{\eta}\right\} \geqslant c(\vec{\eta}, \vec{\eta})
$$



donde $c$ es una constante positiva, para todo posible $\vec{\eta}$. "Parte $G^{\text {s" }}$, representa la expresión del operador $G^{*}$ limitada a algunos sumandos (o todos) según la expresión (3), que recordamos a continuación:

$$
\hat{G}^{s}=\hat{D}^{s}-2 \beta \hat{E}^{s}+2 \alpha i \hat{E}^{a}
$$

"Resto $\hat{G}^{\text {" }}$ representa los. sumandos de la expresión de $\hat{G}^{*}$ no incluidos

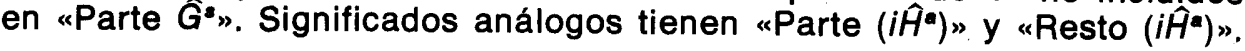

En particular, para el potencial $U=\frac{1}{2}\left(\vec{\eta}, \hat{D}_{0}^{s} \vec{\eta}\right)$, obtenemos las siguientes 
condiciones suficientes de estabilidad:

$$
\begin{gathered}
\left(\vec{\eta}, \hat{D_{0}^{s}} \vec{\eta}\right) \geqslant c(\vec{\eta}, \vec{\eta}) \\
\left.\dot{T}+\dot{U}=-\dot{\vec{\eta}}, 2 \hat{\hat{E}^{s}} \dot{\vec{\eta}}\right)+\operatorname{Re}\left[\vec{\eta},\left(\hat{D}_{1}^{s}+\hat{D}^{a}\right) \vec{\eta}\right] \leqslant 0
\end{gathered}
$$

para un $c>0$ y todo posible $\vec{\eta}$. Este es un resultado conocido, si bien la 2. ${ }^{a}$ desigualdad es difícil de cumplir en la práctica para fuerzas no conservativas.

Si adoptamos el potencial generalizado $U=\frac{1}{2}(\vec{\eta}, \widehat{G} \vec{\eta} \eta)$, de (5) obtenemos el resultado:

$$
\begin{aligned}
& \left.\overrightarrow{[\eta},\left(\hat{D}^{s}+2 \alpha i \hat{E}^{\mathbf{a}}-2 \beta \hat{E}^{s}\right) \vec{\eta}\right] \geqslant c(\vec{\eta}, \vec{\eta}) \\
& {\left[\vec{\eta},\left(\alpha i \hat{D}^{\mathbf{a}}-2 \alpha \beta i \hat{E}^{a}-2 \alpha^{2} \hat{E}^{s}\right) \vec{\eta}\right] \leqslant 0}
\end{aligned}
$$

para un $c>0$ y todo posible $\vec{\eta}$.

Para $\hat{E}=\hat{E}^{a}$ y $\hat{D}=\hat{D}^{s}=\hat{D}_{0}^{s}$, llegamos al resultado del apartado $l$ :

$$
\left(\vec{\eta}, 2 \hat{E}^{a} \vec{\eta}+\hat{D}_{0}^{s} \vec{\eta}\right) \geqslant c(\vec{\eta}, \vec{\eta}), c>0,
$$

todo posible $\vec{\eta}$, con $\beta=0$.

El cálculo operacional desarrollado es de aplicación, en particular, a problemas de estabilidad de sólidos elásticos monodimensionales. Junto con métodos aproximados, como el de Garlekin, se reducen a problemas discretos en dos o tres dimensiones, suficiente en muchos casos en la práctica.

Los resultados del presente trabajo admiten un enfoque distinto considerando los correspondientes problemas de autovalores, con lo que obtenemos un procedimiento más adecuado para las aplicaciones prácticas. Este nuevo planteamiento es objeto de un estudio posterior.

\section{Referencias}

[1] Pars, L. A.: "A treatise on analytical dynamics". Heinemann (1968).

[2] Knops, R. J., Wilkes, E. W.: «Theory of elastic stability". Handbuch der Physik Vol. V 1. ${ }^{2} / 3$. Berlin-Heidelberg-New York (1973).

[3] Dickerson, J. R. and T. K. Gaughey: «Stability of continuous dynamic systems with parametric excitation', J. of Appl. Mech., 212-216 (1969).

[4] Leipholtz, H.: "Stability theory». New York and London. Academic Press (1970).

[5] Leipholtz, $H$.: “Uber die Anwendung vou Liapunov direkter methode anf stabiliz̈ats probleme kontiunierlicher, nichtkonservativer systeme. Ing. Arch., 357-368. (1970). 